\title{
Saúde cardiovascular e validação do escore autorreferido no Brasil: uma análise da Pesquisa Nacional de Saúde
}

\author{
Cardiovascular health and validation of the self-reported score in \\ Brazil: analysis of the National Health Survey
}

Alexandra Dias Moreira (https://orcid.org/0000-0002-4477-5241) ${ }^{1}$

Crizian Saar Gomes (https://orcid.org/0000-0001-6586-4561) ${ }^{1}$

Ísis Eloah Machado (https://orcid.org/0000-0002-4678-2074) ${ }^{2}$

Deborah Carvalho Malta (https://orcid.org/0000-0002-8214-5734) ${ }^{1}$

Mariana Santos Felisbino-Mendes (https://orcid.org/0000-0001-5321-5708) ${ }^{1}$
${ }^{1}$ Escola de Enfermagem, Universidade Federal de Minas Gerais. Av. Alfredo Balena 190, Santa Efigênia. 30130-100 Belo Horizonte MG Brasil. alexandradm84@gmail.com

${ }^{2}$ Escola de Medicina,

Universidade Federal de

Ouro Preto. Ouro Preto MG Brasil.

\begin{abstract}
This paper aims to estimate the prevalence of cardiovascular health and the validity of the Brazilian population's self-reported score. This is a cross-sectional, methodological study with 8,943 individual adults and laboratory data from the 2013 National Health Survey. We employed behavioral (body mass index, tobacco use, diet, physical activity, ideal if $\geq 3$ ideal factors), biological (tobacco use, dyslipidemia, hypertension, and diabetes, ideal if $\geq 3$ ideal factors), and cardiovascular health scores (all factors, ideal if $\geq 4$ ideal factors). Prevalence of sensitivity and specificity scores and analyses of the self-reported scores were estimated, considering the scores with measured variables as the gold standard. Approximately $56.7 \%$ of individuals had ideal values for the measured cardiovascular health score. Sensitivity was $92 \%$ and specificity $30 \%$ for the self-reported biological score. Sensitivity and specificity scores were, respectively, $90.6 \%$ and $97.2 \%$ for self-reported behavior. The self-reported cardiovascular health score had a sensitivity of $92.4 \%$ and specificity of $48.5 \%$. A little over half of the population had an ideal cardiovascular health score. The self-reported score showed good sensitivity and lower proportions of specificity.
\end{abstract}

Key words Validation study, Cardiovascular diseases, Self-report, Surveys and questionnaires
Resumo O objetivo deste artigo é estimar a prevalência de saúde cardiovascular e a validade do escore autorreferido na população brasileira. Estudo transversal, metodológico, com 8.943 indivíduos adultos e dados laboratoriais da Pesquisa Nacional de Saúde 2013. Escores utilizados: comportamental (indice de massa corporal, tabagismo, dieta, atividade fisica, ideal se $\geq 3$ fatores ideais), biológico (tabagismo, dislipidemia, hipertensão e diabetes, ideal se $\geq 3$ fatores ideais) e saúde cardiovascular (todos os fatores, ideal se $\geq 4$ fatores ideais). Estimaram-se prevalências dos escores e análises de sensibilidade e especificidade dos escores autorreferidos, considerando padrão -ouro os escores com variáveis aferidas. Apresentaram valores ideais para o escore de saúde cardiovascular $56,7 \%$ dos indivíduos aferidos. Para o escore biológico autorreferido, a sensibilidade foi de $92 \%$ e a especificidade $30 \%$. Para o comportamental autorreferido, a sensibilidade e a especifcidade foram, respectivamente, $90,6 \%$ e $97,2 \%$. O escore de saúde cardiovascular autorreferido teve sensibilidade $92,4 \%$ e especificidade $48,5 \%$. Pouco mais da metade da população apresentou escore de saúde cardiovascular ideal. O escore autorreferido apresentou boa sensibilidade e menores proporções de especificidade.

Palavras-chave Estudo de validação, Doenças cardiovasculares, Autorrelato, Inquéritos e questionários 


\section{Introdução}

As medidas autorreferidas têm sido amplamente utilizadas em inquéritos de saúde de amplitude nacional para avaliar e monitorar a ocorrência de doenças crônicas não transmissíveis (DCNT) e seus fatores de risco ${ }^{1}$.

Esta metodologia permite a obtenção de informações com maior facilidade operacional e logística, maior agilidade e menor custo $^{1,2} \mathrm{e}$, portanto, muitas vezes é a ferramenta mais importante e conveniente para o monitoramento e a vigilância de doenças e fatores de risco em larga escala na população. No entanto, argumentos contra a confiabilidade dos dados autorreferidos são frequentes e estudos de concordância dessas informações são importantes para demonstrar o quanto essas medidas podem e devem ser consideradas, no sentido de evitar erros de classificação e garantir a qualidade dos dados.

Vários estudos que avaliaram a confiabilidade de doenças crônicas e seus fatores de risco autorreferidos, como o índice de massa corporal (IMC) $)^{3}$, a atividade física ${ }^{4,5}$, a alimentação ${ }^{6}$, a hipertensão ${ }^{7}$ e a diabetes ${ }^{8}$, indicam resultados satisfatórios nas análises de reprodutibilidade e validade, quando as medidas autorreferidas são comparadas com as aferidas. Cabe acrescentar que esses estudos avaliaram os fatores e as doenças de maneira isolada, sendo ainda necessárias pesquisas que avaliem o efeito da utilização das medidas autorreferidas em constructos que analisem a saúde cardiovascular, como o proposto pela American Heart Association (AHA) ${ }^{9}$.

O constructo de saúde cardiovascular ideal, adotado pela American Heart Association (AHA), se baseia principalmente na prevenção primordial como estratégia de prevenção das doenças cardiovasculares (DCV). O critério proposto utiliza um escore composto por sete fatores, incluindo quatro comportamentais [tabagismo, atividade física, índice de massa corporal (IMC) e alimentação] e três biológicos (pressão arterial, glicemia e níveis de colesterol) ${ }^{9}$. Vários estudos têm mostrado que o maior número de fatores em nível ideal está associado ao menor risco de eventos cardiovasculares ${ }^{10-13}$, o que reforça a importância dessas métricas e sua utilização em nível individual e populacional para a proposição de ações voltadas para a prevenção de doenças e de agravos não transmissíveis, como as cardiovasculares, atualmente a maior causa de mortes, e de Disability-Adjusted Life Year (DALY) no nosso país ${ }^{14}$.
Destaca-se que a concordância entre as medidas aferidas e autorreferidas pode ser diferente segundo as características sociodemográficas, econômicas e socioculturais, como sexo, idade, escolaridade e renda, limitando a inferência dos resultados em grupos específicos ${ }^{4,15-17}$.

Assim, o objetivo do presente estudo foi estimar a prevalência de saúde cardiovascular na população brasileira e analisar a validade do escore autorreferido de saúde cardiovascular de acordo com características sociodemográficas.

\section{Materiais e Métodos}

\section{Desenho do estudo}

Trata-se de estudo transversal e metodológico que utilizou os dados da Pesquisa Nacional de Saúde (PNS) de 2013, incluindo os dados laboratoriais coletados em 2014 e 2015, conduzida pelo Instituto de Brasileiro Geografia e Estatística (IBGE) em parceria com o Ministério da Saúde ${ }^{18}$. A PNS é um inquérito nacional de base domiciliar, que utiliza plano amostral por conglomerados em três estágios: setores censitários (unidades primárias); domicílios (unidades secundárias); adultos com idade igual ou maior a 18 anos (unidades terciárias). A metodologia completa e específica desse importante inquérito de saúde populacional está disponível em publicações anteriores ${ }^{19,20}$.

A PNS 2013 contou com 60.202 entrevistas e, em uma subamostra, foi coletado material biológico para análises bioquímicas. A subamostra foi planejada para incluir $25 \%$ da amostra de entrevistados (aproximadamente 15.688 indivíduos), no entanto, a amostra final de indivíduos que tiveram material biológico coletado foi constituída de 8.943 indivíduos. Entre as causas das perdas, citam-se dificuldade na localização do domicílio, recusas dos participantes em coletar o material biológico, desconhecimento do projeto e dos objetivos, e indisponibilidade de horário para atender ao protocolo da pesquisa e também ocorrência de amostras inadequadas para análise.

Destaca-se que para este estudo foram necessárias respostas válidas para todos os componentes aferidos e autorreferidos das variáveis que integram o escore de saúde cardiovascular. Assim, a amostra final para os escores biológicos, comportamental e cardiovascular foi, respectivamente, 6.621, 5.893 e 4.585 indivíduos, devido a dados faltantes aferidos (dados antropométricos e laboratoriais) e autorreferidos. 


\section{Avaliação da saúde cardiovascular}

Para avaliar a saúde cardiovascular foi utilizado o escore de saúde cardiovascular proposto pela $\mathrm{AHA}^{9}$. Destaca-se que o escore pode ser agrupado em: comportamental; biológico e de saúde cardiovascular, e que cada fator pode ser classificado como ideal, intermediário e ruim. As perguntas autorreferidas da PNS permitem discriminar entre ideal e ruim/intermediário, logo, para fins deste estudo, categorizamos dessa forma tanto as medidas aferidas quanto as autorreferidas. Além disso, destaca-se que o fator tabagismo se repete tanto no escore comportamental quanto no biológico devido à importância da cessação do mesmo para a promoção da saúde 9 .

No Quadro 1, são apresentados os pontos de corte e as categorizações utilizadas neste estudo para medidas aferidas e autorreferidas dos escores biológico, comportamental e cardiovascular, conforme proposto por Lloyd-Jones et al. ${ }^{9}$. Ao final, esses escores foram categorizados em ideal, se $\geq 3$ fatores para os escores biológico e comportamental, e ruim/intermediário, no caso de $<$ 3 fatores em nível ideal. Para o escore de saúde cardiovascular, as pessoas com valores $\geq 4$ fatores foram classificadas como portadoras de escore ideal e aquelas com valores $<4$ foram incluídas na categoria de ruim/intermediário.

As informações autorreferidas foram obtidas a partir do questionário da PNS 2013. O tabagismo foi avaliado por meio das perguntas: "Atualmente, o(a) sr(a) fuma algum produto do tabaco?", "E no passado, o(a) sr(a) fumou algum produto do tabaco?" e "P59. Há quanto tempo o(a) $\operatorname{sr}($ a) parou de fumar?".

A dieta foi avaliada a partir de quatro, dos cinco critérios da proposta original, a saber: consumo regular de frutas e hortaliças, de peixes, de refrigerantes e bebidas açucaradas e de sal, conforme descrito em estudo anterior ${ }^{21}$. O consumo de grãos integrais, que compõe o indicador de dieta no escore original, não foi avaliado, por não ter sido incluído no inquérito. A avaliação do consumo de hortaliças e frutas se deu pelas perguntas: "Em quantos dias da semana, o(a) sr(a) costuma comer salada de alface e tomate ou salada de qualquer outra verdura ou legume cru?" "Em geral, quantas vezes por dia o(a) $\operatorname{sr}(a)$ come este tipo de salada?"; "Em quantos dias da semana o(a) sr(a) costuma comer frutas?"; e "Em geral, quantas vezes por dia o(a) sr(a) come frutas?”. O consumo de peixes foi avaliado pela pergunta "Em quantos dias da semana o(a) sr(a) costuma comer peixe?". O consumo de refrige- rante e sucos artificiais foi avaliado pelas perguntas: "Em quantos dias da semana o(a) sr(a) costuma tomar refrigerante (ou suco artificial)?” e "Em geral, quantos copos de refrigerante ou suco artificial o(a) sr(a) costuma tomar por dia?”.

A prática de pelo menos 150 minutos de atividades físicas por semana de atividades moderadas ou 75 minutos por semana de atividades vigorosas no lazer foi avaliada pelas perguntas: "Quantos dias por semana o(a) sr(a) costuma praticar exercício físico ou esporte?"; "Em geral, no dia que o(a) sr(a) pratica exercício ou esporte, quanto tempo dura esta atividade?". Foram consideradas vigorosas atividades como corrida, ginástica aeróbica, futebol e basquetebol.

As medidas autorreferidas de peso e altura avaliadas neste estudo foram informadas durante a entrevista pelas seguintes perguntas: “ $\mathrm{O}(\mathrm{a})$ sr.(a) sabe seu peso (mesmo que seja valor aproximado)?" "O(a) sr.(a) sabe sua altura? (mesmo que seja valor aproximado). Para mensuração direta dos parâmetros antropométricos foi seguida as recomendações da Organização Mundial da Saúde $(\mathrm{OMS})^{22}$. Para aferição do peso, foi utilizada balança digital com capacidade de 150 quilos e precisão de 100 gramas e, para aferição da altura, estadiômetro portátil. Por meio do peso e da estatura, foi calculado o IMC utilizando-se a fórmula: $\mathrm{IMC}=$ peso em $\mathrm{kg}$ / (altura em metros * altura em metros). A mesma fórmula foi utilizada com as medidas obtidas pelo método autorreferido e por medição direta.

Para a avaliação autorreferida de hipertensão, utilizou-se a pergunta: "Algum médico já lhe deu o diagnóstico de hipertensão arterial (pressão alta)?”. A medição direta da pressão arterial foi realizada a partir do método oscilométrico com uso do aparelho automático, garantindo que, por no mínimo 30 minutos antes da medida de pressão arterial, o indivíduo não fumasse, não se alimentasse e não fizesse consumo de nenhum tipo de bebida, exceto água, além de não ter realizado exercícios físicos na última uma hora ${ }^{22}$.

O colesterol alterado foi avaliado pelo método autorreferido pela pergunta "Algum médico já lhe deu o diagnóstico de colesterol alto?”. Para a medição direta a coleta de sangue periférico foi realizada com dispensa de jejum em tubos com gel. Aguardaram-se 30 minutos para a retração do coágulo e posteriormente foi realizada centrifugação e encaminhamento das amostras sob refrigeração de 2 a $8^{\circ} \mathrm{C}$, com controle da temperatura nas diversas etapas. $\mathrm{O}$ colesterol total foi dosado por método enzimático/colorimétrico automatizado $^{23}$. 
Quadro 1. Classificação dos escores comportamental, biológico e de saúde cardiovascular em ruim/intermediário e ideal de acordo com os dados autorreferidos e aferidos pela Pesquisa Nacional de Saúde, 2013.

\begin{tabular}{|c|c|c|c|c|}
\hline \multirow{2}{*}{ Componentes e Escores } & \multicolumn{2}{|c|}{ Autorreferidos } & \multicolumn{2}{|c|}{ Aferidos } \\
\hline & Pobre/intermediário & Ideal & Pobre/intermediário & Ideal \\
\hline Tabagismo & $\begin{array}{l}\text { Fumante atual ou ex-fumante }<12 \\
\text { meses }\end{array}$ & $\begin{array}{l}\text { Nunca fumou ou parou de fumar } \geq 12 \\
\text { meses }\end{array}$ & $\begin{array}{l}\text { Fumante atual ou ex-fumante }<12 \\
\text { meses }\end{array}$ & $\begin{array}{l}\text { Nunca fumou ou parou de fumar } \geq \\
12 \text { meses }\end{array}$ \\
\hline Dieta & $<4$ componentes ideal $^{\star}$ & 4 componentes ideal ${ }^{\star}$ & $<4$ componentes ideal $^{\star}$ & 4 componentes ideal $^{\star}$ \\
\hline Atividade física & $\begin{array}{l}<150 \mathrm{~min} / \mathrm{sem} \text { atividades } \\
\text { moderadas ou }<75 \mathrm{~min} / \mathrm{sem} \text { de } \\
\text { atividades vigorosas no lazer }\end{array}$ & $\begin{array}{l}\geq 150 \mathrm{~min} / \mathrm{sem} \text { atividades moderadas } \\
\text { ou } \geq 75 \mathrm{~min} / \mathrm{sem} \text { de atividades } \\
\text { vigorosas no lazer }\end{array}$ & $\begin{array}{l}<150 \mathrm{~min} / \mathrm{sem} \text { atividades moderadas } \\
\text { ou }<75 \mathrm{~min} / \mathrm{sem} \text { de atividades } \\
\text { vigorosas no lazer }\end{array}$ & $\begin{array}{l}\geq 150 \mathrm{~min} / \mathrm{sem} \text { atividades moderadas } \\
\text { ou } \geq 75 \mathrm{~min} / \mathrm{sem} \text { de atividades } \\
\text { vigorosas no lazer }\end{array}$ \\
\hline $\begin{array}{l}\text { Índice de Massa Corporal } \\
\text { (IMC) }\end{array}$ & $\begin{array}{l}\mathrm{IMC} \geq 25 \mathrm{Kg} / \mathrm{m}^{2} \text { a partir de peso e } \\
\text { altura autoreferido }\end{array}$ & $\begin{array}{l}\mathrm{IMC}<25 \mathrm{Kg} / \mathrm{m}^{2} \text { a partir de peso e } \\
\text { altura autoreferido }\end{array}$ & $\begin{array}{l}\mathrm{IMC} \geq 25 \mathrm{Kg} / \mathrm{m}^{2} \text { a partir de peso e } \\
\text { altura aferidos }\end{array}$ & $\begin{array}{l}\mathrm{IMC}<25 \mathrm{Kg} / \mathrm{m}^{2} \text { a partir de peso e } \\
\text { altura aferidos }\end{array}$ \\
\hline Escore comportamental & \multicolumn{2}{|c|}{$\begin{array}{c}\text { Soma dos } 4 \text { fatores anteriores } \\
\text { Ideal } \geq 3 \text { fatores }\end{array}$} & \multicolumn{2}{|c|}{$\begin{array}{c}\text { Soma dos } 4 \text { fatores anteriores } \\
\text { Ideal } \geq 3 \text { fatores } \\
\end{array}$} \\
\hline Pressão Arterial & $\begin{array}{l}\text { Diagnóstico médico ou } \\
\text { medicamento para hipertensão } \\
\text { arterial }\end{array}$ & $\begin{array}{l}\text { Ausência de diagnóstico ou } \\
\text { medicamento para hipertensão arterial }\end{array}$ & $\begin{array}{l}\mathrm{PAS} \geq 120 \mathrm{mmHg} \text { ou } \mathrm{PAD} \geq 80 \mathrm{mmHg} \\
\text { ou uso de medicação para PA }\end{array}$ & $\begin{array}{l}\text { PAS }<120 \mathrm{mmHg} \text { ou PAD }<80 \mathrm{mmHg} \\
\text { ou uso de medicação para PA }\end{array}$ \\
\hline Colesterol total & $\begin{array}{l}\text { Diagnóstico médico de colesterol } \\
\text { aumentado }\end{array}$ & $\begin{array}{l}\text { Ausência de diagnóstico médico de } \\
\text { colesterol aumentado }\end{array}$ & $\mathrm{CT} \geq 200 \mathrm{mg} / \mathrm{dL}$ & $\mathrm{CT}<200 \mathrm{mg} / \mathrm{dL}$ \\
\hline Diabetes & $\begin{array}{l}\text { Diagnóstico ou medicamento para } \\
\text { diabetes }\end{array}$ & $\begin{array}{l}\text { Ausência de diagnóstico ou } \\
\text { medicamento para diabetes }\end{array}$ & $\begin{array}{l}\text { Hemoglobina glicada } \geq 5.6 \text { ou uso de } \\
\text { medicação para diabetes }\end{array}$ & $\begin{array}{l}\text { Hemoglobina glicada } \geq 5.6 \text { ou uso de } \\
\text { medicação para diabetes }\end{array}$ \\
\hline Escore biológico & \multicolumn{2}{|c|}{$\begin{array}{l}\text { Soma dos } 3 \text { fatores anteriores mais o tabagismo } \\
\text { Ideal } \geq 3 \text { fatores }\end{array}$} & \multicolumn{2}{|c|}{$\begin{array}{l}\text { Soma dos } 3 \text { fatores anteriores mais o tabagismo } \\
\text { Ideal } \geq 3 \text { fatores }\end{array}$} \\
\hline Saúde Cardiovascular Final & \multicolumn{2}{|c|}{$\begin{array}{l}\text { Soma dos } 7 \text { fatores } \\
\text { Ideal } \geq 4 \text { fatores }\end{array}$} & \multicolumn{2}{|c|}{$\begin{array}{l}\text { Soma dos } 7 \text { fatores } \\
\text { Ideal } \geq 4 \text { fatores }\end{array}$} \\
\hline
\end{tabular}

Nota: Classificação realizada conforme critérios American Heart Association (AHA) ${ }^{9} ;{ }^{*}$ Componentes ideal: 5 porções de frutas, verduras e legumes $5 \mathrm{x}$ semana; consumo de peixe 2 vezes na semana; não consumir refrigerante; consumir menos de $5 \mathrm{~g}$ de sal por dia. PAS: Pressão Arterial Sistólica; PAD: Pressão Arterial Diastólica; CT: Colesterol Total 
Para a avaliação de diabetes, foram utilizadas as perguntas: "Algum médico já lhe deu o diagnóstico de diabetes?" e "Nas duas últimas semanas, por causa do diabetes, o(a) $\operatorname{sr}(\mathrm{a})$ : a. tomou medicamentos orais para baixar o açúcar? b. Usou insulina?". O critério medido para diabetes foi a hemoglobina glicada, com coleta de sangue periférico realizada a qualquer hora do dia, sem jejum. O sangue foi colhido em tubo com ácido etilenodiamino tetra-acético (EDTA), enquanto que a hemoglobina glicada foi dosada por cromatografia líquida de alta performance por troca iônica (high pressure liquid chromatography HPLC) ${ }^{23}$.

\section{Análise dos dados}

$\mathrm{Na}$ análise dos dados, foram apresentadas frequências e médias $( \pm \mathrm{DP})$ de variáveis sociodemográficas dos indivíduos e calculadas as prevalências e os intervalos de 95\% de Confiança (IC95\%) dos componentes individuais, bem como dos escores comportamental, biológico e de saúde cardiovascular aferidos e autorreferidos. A validade dos escores biológico, comportamental e de saúde cardiovascular autorreferidos foi avaliada utilizando cálculo de sensibilidade e especificidade, com os respectivos IC95\%, tendo como padrão-ouro as medidas aferidas baseadas em dados antropométricos e laboratoriais. A sensibilidade foi calculada como (verdadeiros positivos) / (verdadeiros positivos + falsos negativos) e a especificidade como (verdadeiros negativos) / (verdadeiros negativos + falsos positivos). Adicionalmente, calculamos os valores preditivos positivos (VPP) - verdadeiros positivos / (verdadeiros positivos + falsos positivos) e negativos (VPN) - verdadeiros negativos / (verdadeiros negativos + falsos negativos) para todos os escores. Todas as análises foram estratificadas por sexo (masculino, feminino), faixa etária (18-49 anos; 50-59 anos e 60 ou mais) e nível de escolaridade (até 8 anos de estudo; de 9 a 11 anos de estudo; e 12 anos ou mais de estudo).

As análises foram conduzidas utilizando-se o software Stata 14.0, sendo considerados o efeito complexo da amostragem e o desenho do estudo.

\section{Considerações éticas}

A PNS foi aprovada pela Comissão Nacional de Ética em Pesquisa (CONEP) do Conselho Nacional de Saúde (CNS), Ministério da Saúde. A participação do adulto na pesquisa foi voluntá- ria e a confidencialidade das informações foi garantida. Os participantes da pesquisa assinaram o Termo de Consentimento Livre e Esclarecido (TCLE) e autorizaram a coleta de exames laboratoriais.

\section{Resultados}

Dos 8.943 indivíduos que tiveram os dados autorreferidos, antropométricos e laboratoriais coletados, 51,2\% (IC95\% 51,5 - 54,3\%) eram do sexo feminino e 49,3\% (IC95\% 47,9 - 50,7\%) relataram até 8 anos de estudo. A média de idade dos participantes deste estudo foi de 43,2 anos \pm 23,7 DP (dados não mostrados).

As prevalências dos escores e das variáveis que os compõem para medidas autorreferidas e aferidas são apresentadas na Tabela 1 . Em relação aos escores aferidos, 65,2 \% (IC95\%: 63,7-66,7\%), 17,3\% (IC95\%: 16,0-18,6\%), e 56,7\% (IC95\%: $54,8-58,6 \%)$ da população apresentaram valores ideais para os escores biológico, comportamental e de saúde cardiovascular, respectivamente. Ao avaliar as medidas autorreferidas, foram classificados com escore biológico ideal 84,4\% (IC95\%: $83,2-85,4 \%$ ), escore comportamental ideal $17,9 \%$ (IC95\%: 16,6-19,3\%) e escore de saúde cardiovascular ideal 74,7 \% (IC95\%: 73,0-76,3\%) dos indivíduos.

Em relação às análises de sensibilidade dos escores biológico, comportamental e de saúde cardiovascular, de maneira geral, os valores variaram de $72,9 \%$ a $98 \%$ para o total de indivíduos e ao estratificar por sexo, idade e escolaridade. Por outro lado, ao avaliar as especificidades para o total de indivíduos e estratificadas por variáveis sociodemográficas, houve maior variabilidade, com menores valores para o escore biológico (entre 17,1 e $39,6 \%$ ) e maiores valores para o escore comportamental (96,4 e 98,1\%). Ressalta-se que em relação às variáveis que compõem o escore comportamental há dados aferidos somente para o IMC, o que possibilitou sua diferenciação no escore autorreferido e aferido. As demais variáveis (prática de atividade física, tabagismo e dieta) estão disponíveis apenas como autorreferidas na PNS. Para o escore de saúde cardiovascular, foram encontrados resultados entre 36,8 a 59,8\% de especificidade. Os VPPs e VPNs para o escore de saúde cardiovascular foram, respectivamente, $70,1 \%$ e 83,0 . Todos os resultados de sensibilidade, especificidade, VPP e VPN estão descritos na Tabela 2. 


\begin{tabular}{|c|c|c|c|}
\hline \multirow[t]{35}{*}{ 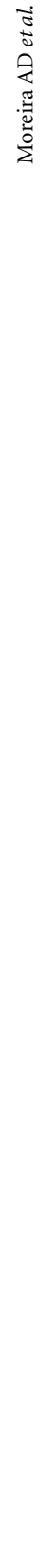 } & $\begin{array}{l}\text { Tabela 1. Prevalência da saúde cardiovascular da } \\
\text { aferidos e autorreferidos. PNS } 2013 \text {. }\end{array}$ & sileira comparando & es individuais e escores \\
\hline & \multirow{2}{*}{ Métricas de saúde cardiovascular } & Autorreferido & Aferido \\
\hline & & $\%(\mathrm{IC} 95 \%)$ & $\%(\mathrm{IC} 95 \%)$ \\
\hline & \multicolumn{3}{|l|}{ Fatores Biológicos } \\
\hline & Colesterol total & $\mathrm{n}=7.560$ & $\mathrm{n}=8.525$ \\
\hline & Ruim/Intermediário & $15,5(14,5-16,5)$ & $32,8(31,5-34,1)$ \\
\hline & Ideal & $84,5(83,5-85,5)$ & $67,3(65,9-68,5)$ \\
\hline & Diabetes & $\mathrm{n}=7.824$ & $\mathrm{n}=8.551$ \\
\hline & Ruim/Intermediário & $7,5(6,8-8,3)$ & $24,2(23,0-25,4)$ \\
\hline & Ideal & $92,5(91,7-93,2)$ & $75,8(74,6-77,0)$ \\
\hline & Pressão arterial & $\mathrm{n}=8.524$ & $\mathrm{n}=8.857$ \\
\hline & Ruim/Intermediário & $23,9(22,8-25,1)$ & $38,8(37,4-40,2)$ \\
\hline & Ideal & $76,1(74,9-77,2)$ & $61,2(59,8-62,6)$ \\
\hline & \multicolumn{3}{|l|}{ Escore Biológico $(n=6.621)$} \\
\hline & Ruim/Intermediário (2-1 fatores) & $15,7(14,6-16,8)$ & $34,8(33,3-36,3)$ \\
\hline & Ideal (4-3 fatores) & $84,4(83,2-85,4)$ & $65,2(63,7-66,7)$ \\
\hline & \multicolumn{3}{|l|}{ Fatores Comportamentais } \\
\hline & \multicolumn{3}{|l|}{ Tabagismo $(\mathrm{n}=8.942)$} \\
\hline & Ruim/Intermediário & $15,9(14,9-17,0)$ & $15,9(14,9-17,0)$ \\
\hline & Ideal & $84,1(83,0-85,1)$ & $84,1(83,0-85,1)$ \\
\hline & \multicolumn{3}{|l|}{ Atividade física no lazer $(\mathrm{n}=8.938)$} \\
\hline & Ruim/Intermediário & $78,3(77,0-79,4)$ & $78,3(77,0-79,4)$ \\
\hline & Ideal & $21,8(20,6-23,0)$ & $21,8(20,6-23,0)$ \\
\hline & Índice de massa corporal & $\mathrm{n}=5.960$ & $\mathrm{n}=8.853$ \\
\hline & Ruim/Intermediário & $54,6(52,9-56,3)$ & $57,3(55,9-58,7)$ \\
\hline & Ideal & $45,4(43,7-47,1)$ & $42,7(41,3-44,1)$ \\
\hline & \multicolumn{3}{|l|}{ Alimentação $(\mathrm{n}=8.942)$} \\
\hline & Ruim/Intermediário & $80,6(79,6-81,6)$ & $80,6(79,6-81,6)$ \\
\hline & Ideal & $19,4(18,4-20,4)$ & $19,4(18,4-20,4)$ \\
\hline & \multicolumn{3}{|l|}{ Escore Comportamental $(n=5.893)$} \\
\hline & Ruim/Intermediário (2-1 fatores) & $82,1(80,7-83,4)$ & $82,8(81,4-84,4)$ \\
\hline & Ideal (4-3 fatores) & $17,9(16,6-19,3)$ & $17,3(16,0-18,6)$ \\
\hline & \multicolumn{3}{|l|}{ Escore de saúde cardiovascular $(\mathrm{n}=4.585)$} \\
\hline & Ruim/Intermediário & $25,3(23,7-27,0)$ & $43,3(41,4-45,2)$ \\
\hline & Ideal & $74,7(73,0-76,3)$ & $56,7(54,8-58,6)$ \\
\hline
\end{tabular}

\section{Discussão}

Na população brasileira, pouco mais da metade dos indivíduos possui escore de saúde cardiovascular ideal, considerando as medidas objetivas de pressão arterial, hemoglobina glicada e IMC. Estudos demonstraram que indivíduos que não possuem resultados ideais para esse escore apresentam maior risco de desenvolverem eventos cardiovasculares $^{24}$ e outros agravos, como o declínio da função cognitiva ${ }^{25,26}$, a doença renal ${ }^{27} \mathrm{e}$ menor qualidade de vida ${ }^{28}$. Os achados deste estudo apontam que grande parte dos brasileiros possuem alterações metabólicas e hábitos de vida desfavoráveis que podem influenciar negativamente as taxas de morbimortalidade por doenças crônicas.

Em relação ao escore de saúde cardiovascular, em geral, observaram-se altas proporções de sensibilidade e baixas especificidades nas análises de validação. As menores especificidades desse escore, que combina os comportamentais e os biológicos, devem-se, majoritariamente, àquelas encontradas para o escore biológico. Isso significa que as pessoas que apresentam saúde ideal avaliadas por aferições objetivas (padrão-ouro) 
Tabela 2. Análises de sensibilidade, especificidade, valores preditivos positivos (VPP) e negativos (VPN) dos escores biológico, comportamental e de saúde cardiovascular, estratificadas por sexo, idade e escolaridade. PNS 2013.

\begin{tabular}{|c|c|c|c|c|}
\hline Escores & $\begin{array}{c}\text { \% Sensibilidade } \\
\text { (IC95\%) }\end{array}$ & $\begin{array}{c}\text { \% Especificidade } \\
\text { (IC95\%) }\end{array}$ & $\begin{array}{c}\text { VPP } \\
\text { (IC 95\%) } \\
\end{array}$ & $\begin{array}{c}\text { VPN } \\
(\text { IC95\%) } \\
\end{array}$ \\
\hline Escore Biológico & $92(90,90-92,9)$ & $30(27,6-32,4)$ & $73,5(71,5-75,4)$ & $64,6(60,2-68,9)$ \\
\hline \multicolumn{5}{|l|}{ Sexo } \\
\hline Feminino & $90,8(89,3-92,0)$ & $33,9(30,8-37,2)$ & $68,5(65,8-70,9)$ & $69,4(63,1-75,1)$ \\
\hline Masculino & $93,5(91,8-94,8)$ & $25,6(22,3-29,2)$ & $80,8(78,8-82,6)$ & $71,8(63,6-78,8)$ \\
\hline \multicolumn{5}{|l|}{ Faixa etária } \\
\hline 18-49 anos & $98,1(97,4-98,6)$ & $17,1(13,8-21,0)$ & $80,8(78,8-82,6)$ & $71,8(63,6-78,8)$ \\
\hline 50 a 59 anos & $87,9(83,8-91,0)$ & $33,7(29,3-38,4)$ & $54,6(50,6-58,6)$ & $75,4(68,1-81,4)$ \\
\hline 60 anos ou mais & $72,9(69,1-76,4)$ & $39,6(35,8-43,5)$ & $53,9(50,5-57,4)$ & $60,2(55,2-64,9)$ \\
\hline \multicolumn{5}{|l|}{ Escolaridade (em anos de estudo) } \\
\hline Até 8 anos & $86,4(84,3-88,2)$ & $33,1(30,2-36,1)$ & $61,9(59,6-64,3)$ & $65,8(61,5-69,9)$ \\
\hline $8-11$ anos & $96,4(95,1-97,3)$ & $24,6(20,2-29,6)$ & $73,5(65,5-80,2)$ & $75,8(73,07-78,5)$ \\
\hline 12 anos ou mais & $94,9(92,7-96,4)$ & $26,1(19,8-33,7)$ & $82,6(79,2-85,6)$ & $58,1(46,4-68,9)$ \\
\hline Escore Comportamental Total & $90,6(87,5-92,9)$ & $97,2(96,5-97,7)$ & $87,1(84,2-89,6)$ & $98,0(97,3-98,5)$ \\
\hline \multicolumn{5}{|l|}{ Sexo } \\
\hline Feminino & $91,6(87,8-94,3)$ & $96,5(95,5-97,3)$ & $83,8(79,4-87,4)$ & $98,3(97,5-98,8)$ \\
\hline Masculino & $89,7(84,7-93,2)$ & $97,9(96,9-98,6)$ & $90,4(86,3-93,4)$ & $97,7(96,5-98,5)$ \\
\hline \multicolumn{5}{|l|}{ Faixa Etária } \\
\hline 18-49 anos & $90,1(86,1-93,1)$ & $97,5(96,6-98,1)$ & $89,5(86,0-92,3)$ & $97,6(96,6-98,4)$ \\
\hline 50 a 59 anos & $93,1(85,6-96,9)$ & $96,7(94,9-97,9)$ & $77,1(66,9-84,8)$ & $99,2(98,2-99,6)$ \\
\hline 60 anos ou mais & $90,9(84,4-94,9)$ & $96,8(95,2-97,9)$ & $85,0(78,2-90,0)$ & $98,2(96,7-98,9)$ \\
\hline \multicolumn{5}{|l|}{ Escolaridade (em anos de estudo) } \\
\hline Até 8 anos & $86(78,8-91,1)$ & $98,1(97,3-98,7)$ & $85,8(80,3-89,9)$ & $98,2(97,1-98,9)$ \\
\hline $8-11$ anos & $89,3(83,9-93,1)$ & $96,4(95,0-97,4)$ & $84,9(79,7-89,0)$ & $97,5(96,1-98,4)$ \\
\hline 12 anos ou mais & $96,1(92,3-98,1)$ & $96,7(94,7-97,9)$ & $90,9(85,8-94,3)$ & $98,6(97,2-99,3)$ \\
\hline Escore Saúde cardiovascular & $92,4(91,0-93,6)$ & $48,5(45,7-51,4)$ & $70,1(68,1-72,1)$ & $83,0(80,1-85,3)$ \\
\hline \multicolumn{5}{|l|}{ Sexo } \\
\hline Feminino & $92,7(90,9-94,2)$ & $51,9(48,0-55,7)$ & $74,2(71,6-76,7)$ & $82,7(78,7-86,1)$ \\
\hline Masculino & $92(89,7-93,9)$ & $45,5(41,4-49,6)$ & $65,8(62,6-68,9)$ & $83,4(78,8-87,1)$ \\
\hline \multicolumn{5}{|l|}{ Faixa etária } \\
\hline 18-49 anos & $96,4(95,1-97,4)$ & $36,8(32,3-41,5)$ & $77,1(74,6-79,4)$ & $82,4(76,6-86,9)$ \\
\hline 50 a 59 anos & $86,5(80,4-90,9)$ & $54,1(48,9-59,2)$ & $53,2(48,0-58,3)$ & $86,9(81,0-91,2)$ \\
\hline 60 anos ou mais & $79,5(74,6-83,7)$ & $59,8(55,0-64,3)$ & $58,0(53,2-62,7)$ & $80,7(76,0-85,7)$ \\
\hline \multicolumn{5}{|l|}{ Escolaridade (em anos de estudo) } \\
\hline Até 8 anos & $87,1(83,8-89,8)$ & $54(50,3-57,6)$ & $58,9(55,5-62,2)$ & $84,7(80,8-87,8)$ \\
\hline $8-11$ anos & $94,2(92,0-95,8)$ & $41(35,9-46,4)$ & $72,5(69,1-75,8)$ & $81,1(74,7-86,2)$ \\
\hline 12 anos ou mais & $95,5(93,5-97,0)$ & $45,1(37,8-52,6)$ & $81,4(77,5-84,7)$ & $80,2(72,1-86,4)$ \\
\hline
\end{tabular}

tendem a se autoclassificarem como saudáveis, contudo, os baixos valores de especificidade refletem baixa concordância de saúde pobre/intermediária aferida e autodeclarada. Ou seja, uma parcela dos indivíduos que declaram componentes do escore de saúde cardiovascular como ideal na verdade apresentaram alterações laboratoriais subclínicas ou subdiagnóstico das doenças e agravos que compõem o escore biológico. Essa lacuna entre as medidas tem sido demonstrada em outros estudos ${ }^{8,29,30}$.

Quanto ao escore comportamental, houve uma menor diferença entre as proporções aferidas e autorreferidas, considerando que, dentre as variáveis que o compõem - atividade física, alimentação, tabagismo e IMC -, apenas a última 
teve aferição objetiva neste estudo, cujas medidas foram validadas em estudo prévio, com resultados aceitáveis ${ }^{3}$.

Em relação ao escore biológico, especificamente, a menor sensibilidade em indivíduos com até 8 anos de estudo em relação àqueles com maior escolaridade pode indicar um menor conhecimento sobre a situação de saúde em pessoas menos instruídas, o que já foi previamente observado em estudo que analisou a autoavaliação da saúde da população brasileira ${ }^{31}$. As maiores especificidades nesse escore foram evidenciadas em mulheres e em pessoas acima de 50 anos, em comparação aos homens e aos adultos mais jovens. Esses achados podem ser reflexo da maior busca dos serviços de saúde para a prevenção e o rastreamento pelas mulheres 8 . Relacionam-se, ainda, a frequente necessidade de acompanhamento periódico dos indivíduos mais velhos por profissionais de saúde, o que contribui para o diagnóstico e o reconhecimento das doenças pelos idosos. A gravidade das doenças tende a ser maior com o aumento da idade, o que poderia diminuir o subdiagnóstico, mediante a presença de sinais e sintomas, enquanto que indivíduos mais jovens, ainda em fase inicial do adoecimento, podem não ter sido diagnosticados ${ }^{15,16}$. Exigese, portanto, cautela na interpretação das respostas em determinados grupos populacionais, cujas condições sociodemográficas podem interferir nos inquéritos ${ }^{16,29,30,32}$.

Em análise adicional deste trabalho, dos indivíduos que declararam não ter hipertensão e diabetes, aproximadamente $20 \%$ apresentaram alterações na aferição da pressão arterial e do exame de hemoglobina glicada (dados não mostrados). Outras pesquisas convergem em direção a esses achados, resultando em altas proporções de subestimação na prevalência de doenças e de fatores de risco cardiovasculares ${ }^{29,30,33}$.

Dessa forma, as medidas autorreferidas podem minimizar a real carga das doenças na população brasileira, por razões relacionadas às deficiências nas políticas públicas, financiamento do sistema de saúde e, consequentemente, ao acesso limitado aos serviços, à prevenção e ao rastreamento ${ }^{8}$. Vale destacar que medidas autorreferidas dependem, ainda, do conhecimento da situação de saúde, da interpretação das perguntas do inquérito e da capacidade dos indivíduos em recordar as informações ${ }^{15,29}$.

Por outro lado, a morbidade referida tem sido utilizada em diversos estudos nacionais s $^{23,30,34}$ e internacionais ${ }^{35,36}$ como forma alternativa de estimar a carga de doenças nas populações ten- do em vista a dificuldade de realizar aferições e medidas bioquímicas em escala populacional. Os dados deste estudo demonstram superestimação da prevalência de saúde cardiovascular ideal na população brasileira e, por outro lado, a subestimação dos níveis de saúde pobre/intermediária. Destaca-se, portanto, que a carga de doenças em inquéritos autorreferidos deve ser interpretada com cautela, principalmente quando se trata de constructos de dados biológicos que podem se alterar de forma assintomática.

Assim, os achados deste estudo também apontam para a necessidade de discussão das barreiras de acesso ao rastreamento e da importância da educação em saúde com o intuito de prevenir as doenças cardiovasculares e, consequentemente, evitar a mortalidade prematura por esses agravos. No contexto do Sistema Único de Saúde (SUS), a Atenção Primária à Saúde (APS) seria o nível de atenção mais apropriado e com maior capilaridade para se investir em melhoria da identificação e do rastreio dos indivíduos potencialmente em risco, contribuindo para a saúde integral.

Uma limitação deste estudo foi que a realização de exames laboratoriais não foi concomitante às entrevistas domiciliares da PNS 2013, dessa forma, podem ter ocorrido mudanças no estado de saúde de alguns indivíduos no período decorrido entre as entrevistas e a coleta das amostras de material biológico ${ }^{20}$. Em segundo lugar, citase a ausência de medidas objetivas de atividade física, alimentação e tabagismo, o que tornou necessária a inclusão dessas variáveis tanto no escore comportamental aferido como no autorreferido. Por outro lado, esta pesquisa possibilitou a composição do escore de saúde cardiovascular ideal com as medidas aferidas de pressão arterial, hemoglobina glicada, colesterol, peso e altura, demonstrando a importância do uso dessas aferições para estimar dados populacionais, uma vez que foram identificadas divergências entre as condições reais e as autodeclaradas de saúde.

A Pesquisa Nacional de Saúde é a primeira pesquisa representativa brasileira a fornecer, além de informações autorreferidas, medidas objetivas sobre o estado de saúde da população. Os dados clínicos e laboratoriais fornecidos pela PNS 2013 permitiram o cálculo do escore biológico do critério da AHA de saúde cardiovascular e os antropométricos, o cálculo do IMC, que é uma das variáveis utilizadas na criação do escore comportamental desse mesmo critério. As análises comparativas entre escore utilizando somente os valores autorreferidos e o escore contendo in- 
dicadores medidos aponta as discrepâncias entre o autorrelato e a real condição de saúde dos indivíduos devido à existência de doenças subclínicas não detectadas. Os achados deste estudo reforçam a importância do investimento em pesquisas em nível nacional que realizem medidas mais precisas do estado de saúde da população, além da necessidade do fortalecimento das ações de promoção da saúde na APS, de modo a garantir a identificação e o acompanhamento de pessoas potencialmente em risco de desenvolvimento de doenças cardiovasculares.

\section{Referências}

1. Brasil. Ministério da Saúde (MS). Pesquisa Nacional de Demografia e Saúde da Criança e da Mulher - PNDS 2006: dimensões do processo reprodutivo e da saúde da criança. Brasília: MS; 2009. (Série G. Estatística e Informação em Saúde)

2. Monteiro CA, Moura EC, Jaime PC, Lucca A, Florindo AA, Figueiredo ICR, Bernal R, Silva NN. Monitoramento de fatores de risco para doenças crônicas por entrevistas telefônicas. Rev Saude Publica 2005; 39(1):47-57.

3. Moreira NF, Luz VG, Moreira CC, Pereira RA, Sichieri R, Ferreira MG, Muraro AP, Rodrigues PRM. Self-reported weight and height are valid measures to determine weight status: results from the Brazilian $\mathrm{Na}-$ tional Health Survey (PNS 2013). Cad Saude Publica 2018 May 10; 34(5):e00063917.

4. Moreira AD, Claro RM, Felisbino-Mendes MS, Velasquez-Melendez G. Validade e reprodutibilidade de inquérito telefônico de atividade física no Brasil. Rev Bras Epidemiol 2017; 20(1):136-146.

5. Monteiro CA, Florindo AA, Claro RM, Moura EC. Validade de indicadores de atividade física e sedentarismo obtidos por inquérito telefônico. Rev Saude Publica 2008; 42(4):575-581.

6. Mendes LL, Campos SF, Malta DC, Bernal RTI, Sá NNBD, Velásquez-Meléndez, G. Validity and reliability of foods and beverages intake obtained by telephone survey in Belo Horizonte, Brazil. Rev Bras Epidemiol 2011; 14(Supl. 1):80-89.

7. Onur I, Velamuri M. The gap between self-reported and objective measures of disease status in India. PLoS ONE 2008; 13(8):e0202786.

8. Najafi F, Moradinazar M, Hamzeh B, Rezaeian S. The reliability of self-reporting chronic diseases: how reliable is the result of population-based cohort studies. J Prev Med Hyg 2019; 60(4):E349-E353.

\section{Colaboradores}

AD Moreira, CS Gomes, IE Machado, DC Malta e MS Felisbino-Mendes participaram igualmente de todas as etapas de elaboração do artigo.

\section{Agradecimentos}

Ministério da Saúde TED 147/2018 (Laboratório da Pesquisa Nacional de Saúde).
9. Lloyd-Jones DM, Hong Y, Labarthe D, Mozaffarian D, Appel LJ, Van Horn L, Greenlund K, Daniels S, Nichol G, Tomaselli GF, Arnett DK, Fonarow GC, Ho PM, Lauer MS, Masoudi FA, Robertson RM, Roger V, Schwamm LH, Sorlie P, Yancy CW, Rosamond WD; American Heart Association Strategic Planning Task Force and Statistics Committee. Defining and setting national goals for cardiovascular health promotion and disease reduction: the American Heart Association's strategic Impact Goal through 2020 and beyond. Circulation 2010; 121(4):586-613.

10. Zhou L, Zhao L, Wu Y, Wu Y, Gao X, Li Y, Mai J, Nie Z, Ou Y, Guo M, Liu X. Ideal cardiovascular health metrics and its association with 20 -year cardiovascular morbidity and mortality in a Chinese population. J Epidemiol Community Health 2018; 72(8):752-758.

11. Folsom AR, Yatsuya H, Nettleton JA, Lutsey PL, Cushman M, Rosamond WD, ARIC Study Investigators. Community prevalence of ideal cardiovascular health, by the American Heart Association definition, and relationship with cardiovascular disease incidence. J Am Coll Cardiol 2011; 57(16):1690-1696.

12. Guo L, Zhang S. Association between ideal cardiovascular health metrics and risk of cardiovascular events or mortality: a meta-analysis of prospective studies. Clin Cardiol 2017; 40(12):1339-1346.

13. Gaye B, Canonico M, Perier MC, Samieri C, Berr C, Dartigues JF, Tzourio C, Elbaz A, Empana JP. Ideal cardiovascular health, mortality, and vascular events in elderly subjects: the Three-City Study. J Am Coll Cardiol 2017; 69(25):3015-3026. 
14. Malta DC, Duncan BB, Schmidt MI, Teixeira R, Ribeiro ALP, Felisbino-Mendes MS, Machado IE, Velasquez-Melendez G, Brant LCC, Silva DAS, Passos VMA, Nascimento BR, Cousin E, Glenn S, Naghavi M. Trends in mortality due to non-communicable diseases in the Brazilian adult population: national and subnational estimates and projections for 2030. Population and Health Metrics. No prelo 2020.

15. Menezes TN, Oliveira ECT. Validity and concordance of self-reported diabetes mellitus by the elderly. Cien Saude Colet 2019; 24(1):27-34.

16. Fontanelli MM, Teixeira JA, Sales CH, Castro MA, Cesar CL, Alves MC, Goldbaum M, Marchioni DM, Fisberg RM. Validation of self-reported diabetes in a representative sample of São Paulo city. Rev Saude Publica 2017; 51:20.

17. Großschädl F, Haditsch B, Stronegger WJ. Validity of self-reported weight and height in Austrian adults: sociodemographic determinants and consequences for the classification of BMI categories. Public Health Nutr 2012; 15(1):20-27

18. Szwarcwald CL, Malta DC, Pereira CA, Vieira ML, Conde WL, Souza Júnior PR, Damacena GN, Azevedo LO, Azevedo E Silva G, Theme Filha MM, Lopes CS, Romero DE, Almeida Wda S, Monteiro CA. National Health Survey in Brazil: design and methodology of application. Cien Saude Colet 2014; 19(2):333-342.

19. Damacena GN, Szwarcwald CL, Malta DC, Souza Júnior PRBD, Vieira MLFP, Pereira CA, MORAIS OL, Silva Júnior JBD. O processo de desenvolvimento da Pesquisa Nacional de Saúde no Brasil, 2013. Epidemiol Serv Saúde 2015; 24(2):197-206.

20. Szwarcwald CL, Malta DC, Souza Júnior PRB, Almeida WDS, Damacena GN, Pereira CA, Rosenfeld LG. Laboratory exams of the National Health Survey: methodology of sampling, data collection and analysis. Rev Bras Epidemiol 2019; 22(Supl. 02):E190004.

21. Velasquez-Melendez G, Felisbino-Mendes MS, Matozinhos FP, Claro R, Gomes CS, Malta DC. Prevalência de saúde cardiovascular ideal na população brasileira -Pesquisa Nacional de Saúde (2013). Rev Bras Epidemiol 2015; 18(Supl. 2):97-108.

22. Instituto Brasileiro de Geografia e Estatística (IBGE). Pesquisa Nacional de Saúde 2013: Manual de Antropometria. Rio de Janeiro: IBGE; 2013.

23. Malta DC, Silva AGD, Tonaco LAB, Freitas MIF, Velasquez-Melendez G. Time trends in morbid obesity prevalence in the Brazilian adult population from 2006 to 2017. Cad Saude Publica 2019; 35(9):e00223518.

24. van Sloten TT, Tafflet M, Périer MC, Dugravot A, Climie RED, Singh-Manoux A, Empana JP. Association of Change in Cardiovascular Risk Factors With Incident Cardiovascular Events. JAMA 2018; 320(17):1793-1804.

25. Crichton GE, Elias MF, Davey A, Alkerwi A. Cardiovascular health and cognitive function: the Maine-Syracuse Longitudinal Study. PLoS One 2014; 9(3):e89317.

26. Sabia S, Fayosse A, Dumurgier J, Schnitzler A, Empana JP, Ebmeier KP, Dugravot A, Kivimäki M, Singh-Manoux A. Association of ideal cardiovascular health at age 50 with incidence of dementia: 25 year follow-up of Whitehall II cohort study. BMJ 2019; 366:14414.
27. Han QL, Wu SL, Liu XX, An SS, Wu YT, Gao JS, Chen SH, Liu XK, Zhang Q, Mao RY, Shang XM, Jonas JB. Ideal cardiovascular health score and incident en$\mathrm{d}$-stage renal disease in a community-based longitudinal cohort study: the Kailuan Study. BMJ 2016; 6(11):e012486.

28. Allen NB, Badon S, Greenlund KJ, Huffman M, Hong Y, Lloyd-Jones DM. The association between cardiovascular health and health-related quality of life and health status measures among U.S. adults: a crosssectional study of the National Health and Nutrition Examination Surveys, 2001-2010. Health Qual Life Outcomes 2015; 13:152.

29. Johnston DW, Propper C, Shields MA. Comparing subjective and objective measures of health: Evidence from hypertension for the income/health gradient. $J$ Health Econ 2009; 28(3):540-552.

30. Gonçalves VSS, Andrade KRC, Carvalho KMB, Silva MT, Pereira MG, Galvão TF. Accuracy of self-reported hypertension: a systematic review and meta-analysis. J Hypertens 2018; 36(5):970-978.

31. Sousa JL, Alencar GO, Antunes JLF, Silva ZP. Marcadores de desigualdade na autoavaliação da saúde de adultos no Brasil, segundo o sexo. Cad Saude Publica 2018; 36(5):e00230318.

32. Vellakkal S, Subramanian SV, Millett C, Basu S, Stuckler D, Ebrahim S. Socioeconomic inequalities in non-communicable diseases prevalence in India: disparities between self-reported diagnoses and standardized measures. PloS one 2013; 8:7.

33. Baker M, Stabile M, Deri C. What do Self-reported, Objective, Measures of Health Measure? Journal of Human Resources 2004; 39(4):1067-1093.

34. Borgo MV, Pimentel EB, Baldo MP, Souza JB, Malta DC, Mill JG. Prevalence of cardiovascular risk factors in the population of Vitória according to data from VIGITEL and the National Health Interview Survey of 2013. Rev Bras Epidemiol 2019; 22:e190015.

35. Cheng YJ, Imperatore G, Geiss LS, Saydah SH, Albright AL, Ali MK, Gregg EW. Trends and Disparities in Cardiovascular Mortality Among U.S. Adults With and Without Self-Reported Diabetes, 1988-2015. Diabetes Care 2018; 41(11):2306-2315.

36. Marques A, Peralta M, Naia A, Loureiro N, de Matos MG. Prevalence of adult overweight and obesity in 20 European countries, 2014. Eur J Public Health 2018; 28(2):295-300.

Artigo apresentado em 17/08/2020

Aprovado em 18/08/2020

Versão final apresentada em 21/08/2020 